\title{
Copy-number variation and protein expression of DOT1L in pancreatic adenocarcinoma as a potential drug target
}

\author{
HEIKE LOESER $^{1}$, DIRK WALDSCHMIDT ${ }^{2}$, FABIAN KUETTING ${ }^{2}$, CARINA HEYDT $^{1}$, THOMAS ZANDER $^{3}$, \\ PATRICK PLUM ${ }^{4}$, HAKAN ALAKUS $^{4}$, REINHARD BUETTNER $^{1}$ and ALEXANDER QUAAS ${ }^{1}$ \\ ${ }^{1}$ Institute of Pathology; Departments of ${ }^{2}$ Gastroenterology and Hepatology, ${ }^{3}$ Oncology and Hematology \\ and ${ }^{4}$ Visceral Surgery; University of Cologne, Gastrointestinal Cancer Group Cologne, D-50937 Cologne, Germany
}

Received October 5, 2016; Accepted February 14, 2017

DOI: $10.3892 / \mathrm{mco} .2017 .1194$

\begin{abstract}
Adenocarcinoma of the pancreas has a poor prognosis. At present, no relevant personalized targets have been identified. Sequencing studies have implicated gene alterations of disruptor of telomeric silencing 1 like histone lysine methyltransferase (DOT1L) in pancreatic adenocarcinoma. DOT1L is part of the histone modification system and catalyzes methylation of H3K79, which is crucial in cell signaling and DNA damage repair. DOT1L is considered to be a target of therapy in mixed lineage leukemia gene-deficient leukemia cases and a potential target in breast carcinoma. The frequencies and importance of DOT1L copy-number variations and their specific correlation with protein expression in adenocarcinoma of the pancreas have yet to be investigated. In the present study, tissue microarrays of 230 resected pancreatic adenocarcinoma cases were constructed. The tumor tissue was analyzed using fluorescence in situ hybridization (FISH) and immunohistochemistry. In total, 10/225 carcinoma cases (4.4\%) analyzed by immunohistochemistry demonstrated intense nuclear protein expression of DOT1L and in 9/224 tumors analyzed using FISH (4.0\%), copy-number variations (CNV) were detectable. No DOT1L amplification was detected in the carcinoma cohort. To the best of our knowledge, the present study describes for the first time the frequency of CNV of DOT1L using the gold standard fluorescence in situ hybridization (FISH) and their specific correlation to the protein expression in adenocarcinomas of the pancreas. Although the positive cases by immunohistochemistry and copy-number variations by FISH were not congruent with each other, the data suggest a potential role for DOT1L in a small subset of pancreatic cancer cases. The significance of the two analysis methods concerning their druggability in pancreatic adenocarcinoma requires further studies.
\end{abstract}

Correspondence to: Dr Heike Loeser, Institute of Pathology, University of Cologne, Gastrointestinal Cancer Group Cologne, Kerpener Strasse 62, D-50937 Cologne, Germany

E-mail: heike.loeser@uk-koeln.de

Key words: DOT1L, pancreatic adenocarcinoma, immunohistochemistry, fluorescence in situ hybridization, copynumber variations, personalized therapy

\section{Introduction}

Gene expression and regulation are based among other things on the posttranslational modification system of histone proteins (1). Disruptor of telomeric silencing 1 like histone lysine methyltransferase (DOT1L) is an evolutionarily conserved histone methyltransferase that, according to the current state of knowledge, exclusively catalyzes the methylation of H3K79 (2,3). In cancer, this methylation is involved in DNA damage repair and tumorigenesis. DOT1L was revealed to be crucial in leukemic transformation and is associated with poor prognosis in breast and colorectal cancer (4-7). Sequencing studies (www.cbioportal.org) demonstrate DOT1L alterations in $\geq 13 \%$ of pancreatic cancer cases. The frequencies of DOT1L copy-number variations determined using gold standard fluorescence in situ hybridization (FISH), and their specific correlation to protein expression, remain to be established.

At present, no relevant personalized treatable target exists in pancreatic adenocarcinoma, thus conventional chemotherapy and radiotherapy is still the standard systemic therapy (8-10). Nevertheless the prognosis of pancreatic adenocarcinoma remains poor, and surgery is the only curative option for patients. However, only $20 \%$ of adenocarcinomas are operably linked to a curative approach at the time of diagnosis $(11,12)$. Targeted therapy strategies are urgently required for this severe form of carcinoma.

In the present study, DOT1L was evaluated as a potential therapeutic target in pancreatic adenocarcinoma. According to the results of genomic studies, immunohistochemical analysis and FISH were performed, hypothesizing that the identification of a subgroup of carcinoma cases may demonstrate a significant DOT1L copy-number variation or amplification.

\section{Patients and methods}

Patients and tumor samples. In the present retrospective study 230 patients with pancreatic adenocarcinoma who underwent surgical pancreatic resections at the University Hospital of Cologne (Cologne, Germany) between January 1999 and December 2014 were analyzed. For tissue microarray analysis (TMA), two tissue cores from various areas of each tumor were obtained and transferred into a TMA recipient 
block. TMA construction was performed as described previously $(13,14)$. Patient characteristics are presented in Table I. In brief, tissue cylinders with a diameter of $1.2 \mathrm{~mm}$ each were punched from selected tumor tissue blocks using a homemade semi-automated precision instrument and inserted into empty recipient paraffin blocks. Four $\mu \mathrm{m}$-thick sections of the resulting TMA blocks were transferred to an adhesive-coated slide system (Instrumedics Inc., Hackensack, NJ, USA). Consecutive sections were used for FISH and immunohistochemistry.

Immunohistochemistry. Immunohistochemistry (IHC) was performed on TMA slides using the primary rabbit polyclonal antibody specific for DOT1L (\#NB100-40845; dilution, 1:50; NovusBiologicals,Ltd.,Cambridge,UK).Immunohistochemical staining was performed using the Ventana BenchMark stainer (Roche Diagnostics GmbH, Mannheim, Germany) according to the manufacturer's protocol with on-slide controls of the appendix. As a secondary antibody the ready-to-use Ventana Detection kit (cat. no. 760-700; OptiView DAB IHC Detection kit; Ventana, Roche Diagnostics GmbH, Mannheim, Germany) for indirect biotin-free detection of primary rabbit antibody was used. DOTL1 exhibits a nuclear staining pattern. DOT1L staining was evaluated manually using light microscopy by two pathologists and discrepant results were resolved by consensus review.

FISH. FISH for evaluation of DOT1L gene copy numbers was performed with the DOT1L/CEN19 Dual Color Probe (Empire Genomics, Buffalo, NY, USA). Three- $\mu$ m tissue sections on SuperFrost Plus slides were mounted by heating at $56^{\circ} \mathrm{C}$, followed by semi-automated deparaffinization protease digestion washing steps (VP2000 processor system, Abbott Molecular, Wiesbaden, Germany). Protease digestion was performed using the ready-to-use FISH pretreatment kit (Vysis IntelliFISH Universal FFPE Tissue Pretreatment Protease; Abbott Molecular, Wiesbaden, Germany). Slides were hybridized at $37^{\circ} \mathrm{C}$ overnight with the FISH probe. The slides were stained with DAPI prior to analysis. Normal tissue including vessels, fibroblasts, or non-tumor lung tissue served as an internal positive control. Cases were only further evaluated if control tissue nuclei exhibited one or two clearly distinct signals of each color. Tumor tissue was scanned for amplification hot spots using x63 objective (DM5500 fluorescent microscope; Leica Microsystems, Inc., Buffalo Grove, IL, USA). If the signals were homogeneously distributed, then random areas were used for counting the signals. Sixty tumor cells in three areas were evaluated by counting green DOT1L and orange centromere 19 (CEN19) signals. The reading strategy followed that of the cMET-FISH probe to evaluate different levels of amplification. Low-level amplification was defined as $\geq 4$ DOT1L signals in $\geq 40 \%$ of cells. Intermediatelevel amplification was defined as $\geq 5$ DOT1L in $\geq 50 \%$ of cells. High-level amplification was defined as a DOT1L/CEN19 quotient of $2.0, \geq 15$ DOT1L signals in $\geq 10 \%$ of cells or an average DOT1L copy number of $\geq 6$.

Procedures were followed as outlined in accordance with ethical standards formulated in the Helsinki Declaration 1975 (and revised in 1983) with pre-approval by the Ethics Committee at the University Hospital (Cologne, Germany; reference number: 09-232).
Table I. Clinicopathological characteristics of 230 pancreatic adenocarcinoma cases.

\begin{tabular}{lr}
\hline Characteristic & No. of patients \\
\hline Gender & \\
Male & 124 \\
Female & 106 \\
Age, years & \\
$<50$ & 51 \\
50-60 & 70 \\
$>70$ & 99 \\
Tumor stage & \\
pT1 & 9 \\
pT2 & 25 \\
pT3 & 190 \\
pT4 & 6 \\
Lymph node metastasis & \\
N0 & 66 \\
N1 & 164 \\
Resection margin status & \\
R0 & \\
R1 & \\
Grading & 148 \\
G1 & 82 \\
G2 & \\
G3 & \\
\hline & \\
& \\
&
\end{tabular}

\section{Results}

Immunohistochemistry. DOT1L immunohistochemistry was performed on TMA slides, including 230 pancreatic carcinomas. Tissue from the appendix vermiformis served as on-slide control. Of the 230 pancreatic carcinomas, 225 were evaluable. Ten carcinomas $(4.4 \%)$ revealed strong nuclear staining (Fig. 1).

FISH. The DOT1L-FISH-signal pattern revealed an even distribution of the signals in the majority of cases. A total of 224 pancreatic adenocarcinomas were able to be analyzed using FISH; there were 9 cases with single tumor cells exhibiting microclusters of $>5$ DOT1L-signals accompanied by elevated CEN-signals, but no amplification according to the evaluation criteria (using common low-, intermediateand high level amplification criteria of MET analyses) was detected (Table II). These elevated DOT1L signals were detected in cases where polysomy was already detected. Polysomy was slightly increased, ranging from 1.55-2.5 average CEN-signals/cell (Fig. 2).

\section{Discussion}

According to the analysis of sequencing data, certain cancer types exhibit DOT1L gene alterations. Concordant with the biology of MET, the present study hypothesized that a significant elevation of gene copy number of DOT1L may correlate 
Table II. Fluorescence in situ hybridization analysis of copy number variations of DOT1L in 9 pancreatic adenocarcinoma cases.

\begin{tabular}{lccccc}
\hline Patient & $\begin{array}{c}\text { Green signals/ } \\
60 \text { cells }\end{array}$ & $\begin{array}{c}\text { Orange signals/ } \\
60 \text { cells }\end{array}$ & $\begin{array}{c}\text { Ratio green: } \\
\text { orange }\end{array}$ & $\begin{array}{c}\text { Average } \\
\text { green signals }\end{array}$ & $\begin{array}{c}\text { Average } \\
\text { orange signals }\end{array}$ \\
\hline 1 & 162 & 93 & 1.74 & 2.7 & 1.55 \\
2 & 174 & 150 & 1.16 & 2.9 & 2.5 \\
3 & 168 & 94 & 1.78 & 2.8 & 1.6 \\
4 & 186 & 126 & 1.48 & 3.1 & 2.1 \\
5 & 176 & 132 & 1.32 & 2.9 & 2.2 \\
6 & 193 & 145 & 1.32 & 3.2 & 2.4 \\
7 & 167 & 127 & 1.3 & 2.8 & 2.0 \\
8 & 158 & 118 & 1.34 & 2.6 & 2.2 \\
9 & 195 & 130 & 1.5 & 3.2 & \\
\hline
\end{tabular}

DOT1L signal is represented as green; CEN19 signal is represented as orange. DOT1L, disruptor of telomeric silencing 1 like histone lysine methyltransferase; CEN19, centromere 19.

A

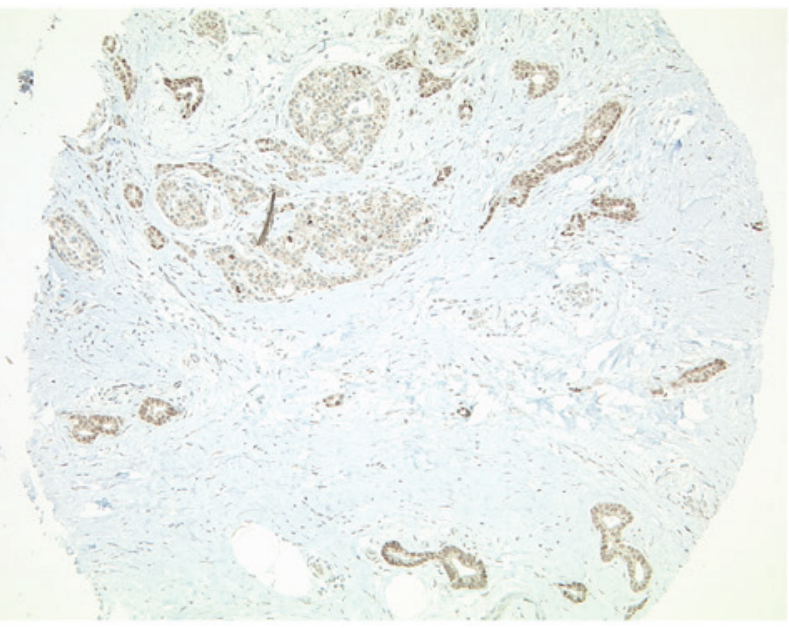

B

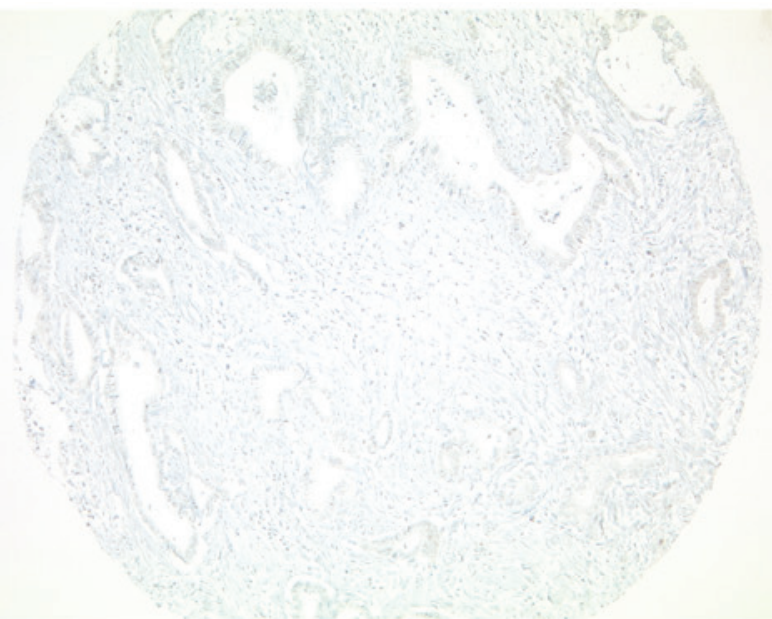

Figure 1. Immunohistochemistry of DOT1L in arrayed pancreatic adenocarcinomas. Nuclear staining for (A) DOT1L (positive) and (B) DOT1L negative tissues. Original magnification, x100. DOT1L, disruptor of telomeric silencing 1 like histone lysine methyltransferase.

with protein overexpression. The augmentation of DOT1L protein expression may further be associated with an increased response to a DOT1L-inhibitor therapy. DOT1L is a druggable target causing cell cycle arrest and leading to chemosensitivity in leukemia $(15,16)$. The DOT1L inhibitor EPZ-5676 is under clinical investigation in a phase I study for mixed lineage leukemia (www.ClinicalTrials.gov; cat. no. NCT01684150). The pharmaco-physiological mechanism of DOTL1 inhibition using the drug EPZ-5676 in mixed lineage leukemia is not fully understood. The maintenance of the MLL-AF6 fusion protein requires $\mathrm{H} 3 \mathrm{~K} 79$ methylation (17). MLL fusion proteins recruit DOT1L, leading to aberrant DOT1L overexpression and H3K79 methylation $(18,19)$. In breast carcinoma, DOT1L is associated with an aggressive phenotype and has also been revealed to be a potential therapeutic target $(4,20,21)$.

Therefore, the present study immunohistochemically evaluated the frequency of pancreatic carcinoma cells that overexpress DOT1L, and how the protein expression correlates with the DOT1L gene copy number. No association between DOT1L gene copy number elevation and protein

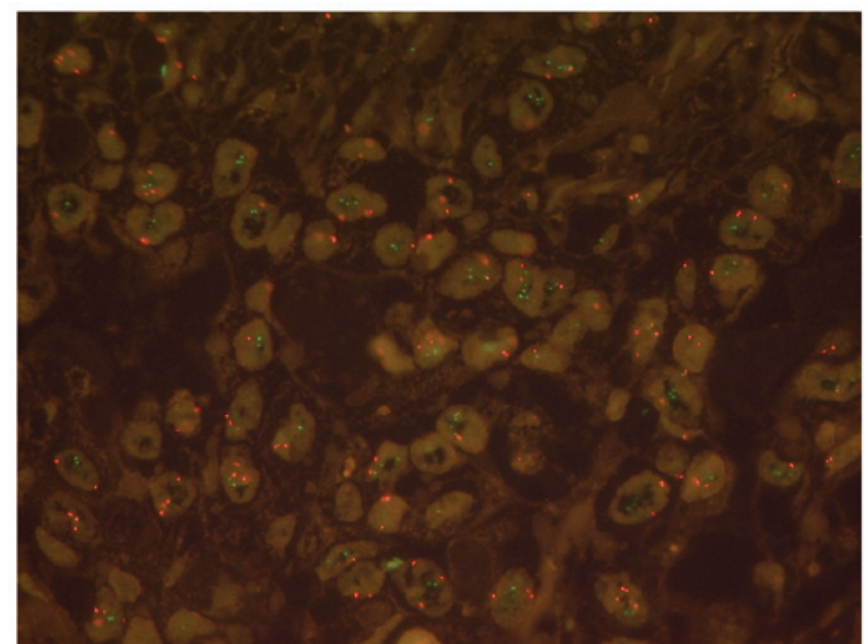

Figure 2. Fluorescence in situ hybridization analysis of DOT1L in arrayed pancreatic adenocarcinoma tissue. Copy number variations of DOT1L are shown as green and the signals of centromere CEN19 presented as orange. DOT1L, disruptor of telomeric silencing 1 like histone lysine methyltransferase; CEN19, centromere 19. 
overexpression in tumor cells of the same cases were identified. Post-transcriptional modifications of DOT1L that inhibit appropriate protein expression may be one explanation. On the other hand, DOT1L protein overexpression (not dependent on an elevation of gene copy number) may be pharmaco-physiologically important if further studies are able to establish the expected association, in which more DOT1L protein be related to more intense methylation of $\mathrm{H} 3 \mathrm{~K} 79$. At present, the methylation of H3K79 is considered to accelerate the activity of various genes involved in cell cycle activity (22). Due to this perception, an inhibition of DOT1L in cancer may be important. Initial evidence suggests that the DOT1L inhibitor EPZ-5676 is effective in mixed lineage leukemia (MLL) $(23,24)$.

According to sequence data, all DOT1L-altered pancreatic carcinoma harbor a KRAS mutation as well (The Cancer Genome Atlas data, www.cbioportal.org). This is not an unexpected finding, due to the fact that $>90 \%$ of all pancreatic carcinomas have KRAS mutations (25). The present study speculates that CNVs of DOT1L in KRAS mutated tumors are likely to be an epiphenomenon. In accordance with this assumption, the KRAS mutation may knock out any therapeutically intended DOT1L inhibition. Furthermore, the distribution of CNVs or protein expression of DOT1L identified in the present study is highly heterogeneous. Tumor cells presenting with CNVs of DOT1L were intermixed with carcinoma cells with regular gene copy numbers. A similar result was observed in immunohistochemically positive tumors: Certain tumor cell groups were DOT1L positive and others were negative.

Inconclusion, a subset of $\sim 4 \%$ of pancreatic adenocarcinomas exhibit CNVs of DOT1L with heterogeneously-distributed single tumor cells showing microclusters of $>5$ DOT1L-signals ( $\leq 14$ signals) or nuclear protein overexpression. None of the tumor samples meets the criteria of amplification. There is no correlation between CNVs and protein overexpression. The significance of possible druggability in pancreatic adenocarcinoma, particularly in the protein positive tumor subgroup, requires further study. However, the present study does not predict a successful therapeutic effect using DOT1L inhibitors in adenocarcinomas of the pancreas.

\section{References}

1. Wang X, Chen CW and Armstrong SA: The role of DOT1L in the maintenance of leukemia gene expression. Curr Opin Genet Dev 36: 68-72, 2016.

2. Lacoste N, Utley RT, Hunter JM, Poirier GG and Côte J: Disruptor of telomeric silencing-1 is a chromatin-specific histone H3 methyltransferase. J Biol Chem 277: 30421-30424, 2002.

3. Farooq Z, Banday S, Pandita TK and Altaf M: The many faces of histone H3K79 methylation. Mutat Res Rev Mutat Res 768: 46-52, 2016.

4. Lee JY and Kong G: DOT1L: A new therapeutic target for aggressive breast cancer. Oncotarget 6: 30451-30452, 2015.

5. Kryczek I, Lin Y, Nagarsheth N, Peng D, Zhao L, Zhao E, Vatan L, Szeliga W, Dou Y, Owens S, et al: IL-22(+)CD4(+) $\mathrm{T}$ cells promote colorectal cancer stemness via STAT3 transcription factor activation and induction of the methyltransferase DOT1L. Immunity 40: 772-784, 2014.

6. Nguyen AT, Taranova O, He J and Zhang Y: DOT1L, the H3K79 methyltransferase, is required for MLL-AF9-mediated leukemogenesis. Blood 117: 6912-6922, 2011.
7. Oktyabri D, Ishimura A, Tange S, Terashima M and Suzuki T: DOT1L histone methyltransferase regulates the expression of BCAT1 and is involved in sphere formation and cell migration of breast cancer cell lines. Biochimie 123: 20-31, 2016.

8. Caparello C, Meijer LL, Garajova I, Falcone A, Le Large TY, Funel N, Kazemier G, Peters GJ, Vasile E and Giovannetti E: FOLFIRINOX and translational studies: Towards personalized therapy in pancreatic cancer. World J Gastroenterol 22: 6987-7005, 2016

9. Takaori K, Bassi C, Biankin A, Brunner TB, Cataldo I, Campbell F, Cunningham D, Falconi M, Frampton AE, Furuse J, et al; IAP/EPC study group on the clinical managements of pancreatic cancer: International Association of Pancreatology (IAP)/European Pancreatic Club (EPC) consensus review of guidelines for the treatment of pancreatic cancer. Pancreatology 16: 14-27, 2016.

10. Gall TM, Tsakok M, Wasan H and Jiao LR: Pancreatic cancer: Current management and treatment strategies. Postgrad Med J 91: 601-607, 2015.

11. Wagner M, Redaelli C, Lietz M, Seiler CA, Friess H and Büchler MW: Curative resection is the single most important factor determining outcome in patients with pancreatic adenocarcinoma. Br J Surg 91: 586-594, 2004.

12. Badger SA, Brant JL, Jones C, McClements J, Loughrey MB, Taylor MA, Diamond T and McKie LD: The role of surgery for pancreatic cancer: A 12-year review of patient outcome. Ulster Med J 79: 70-75, 2010.

13. Simon R, Mirlacher M and Sauter G: Tissue microarrays. Methods Mol Med 114: 257-268, 2005.

14. Helbig D, Ihle MA, Pütz K, Tantcheva-Poor I, Mauch C, Büttner R and Quaas A: Oncogene and therapeutic target analyses in atypical fibroxanthomas and pleomorphic dermal sarcomas. Oncotarget 7: 21763-21774, 2016.

15. Liu W, Deng L, Song Y and Redell M: DOT1L inhibition sensitizes MLL-rearranged AML to chemotherapy. PLoS One 9: e98270, 2014.

16. Stein EM and Tallman MS: Mixed lineage rearranged leukaemia: Pathogenesis and targeting DOT1L. Curr Opin Hematol 22: 92-96, 2015.

17. Deshpande AJ, Chen L, Fazio M, Sinha AU, Bernt KM, Banka D, Dias S, Chang J, Olhava EJ, Daigle SR, et al: Leukemic transformation by the MLL-AF6 fusion oncogene requires the H3K79 methyltransferase Dot11. Blood 121: 2533-2541, 2013.

18. Okada Y, Feng Q, Lin Y, Jiang Q, Li Y, Coffield VM, Su L, Xu G and Zhang Y: hDOT1L links histone methylation to leukemogenesis. Cell 121: 167-178, 2005.

19. Wong M, Polly P and Liu T: The histone methyltransferase DOT1L: Regulatory functions and a cancer therapy target. Am J Cancer Res 5: 2823-2837, 2015.

20. Zhang L, Deng L, Chen F, Yao Y, Wu B, Wei L, Mo Q and Song Y: Inhibition of histone H3K79 methylation selectively inhibits proliferation, self-renewal and metastatic potential of breast cancer. Oncotarget 5: 10665-10677, 2014.

21. Cho MH, Park JH, Choi HJ, Park MK, Won HY, Park YJ, Lee $\mathrm{CH}$, Oh SH, Song YS, Kim HS, et al: DOT1L cooperates with the c-Myc-p300 complex to epigenetically derepress $\mathrm{CDH} 1$ transcription factors in breast cancer progression. Nat Commun 6: 7821, 2015.

22. Kim W, Choi M and Kim JE: The histone methyltransferase Dot1/ DOT1L as a critical regulator of the cell cycle. Cell Cycle 13: 726-738, 2014

23. Daigle SR, Olhava EJ, Therkelsen CA, Basavapathruni A, Jin L, Boriack-Sjodin PA, Allain CJ, Klaus CR, Raimondi A, Scott MP, et al: Potent inhibition of DOT1L as treatment of MLL-fusion leukemia. Blood 122: 1017-1025, 2013.

24. Klaus CR, Iwanowicz D, Johnston D, Campbell CA, Smith JJ, Moyer MP, Copeland RA, Olhava EJ, Scott MP, Pollock RM, et al: DOT1L inhibitor EPZ-5676 displays synergistic antiproliferative activity in combination with standard of care drugs and hypomethylating agents in MLL-rearranged leukemia cells. J Pharmacol Exp Ther 350: 646-656, 2014.

25. Morris JP IV, Wang SC and Hebrok M: KRAS, Hedgehog, Wnt and the twisted developmental biology of pancreatic ductal adenocarcinoma. Nat Rev Cancer 10: 683-695, 2010. 